\title{
Método práctico y tiempo de intubación endotraqueal entre alumnos de la carrera de médico cirujano
}

\author{
Rulli Russi-Santes ${ }^{1,2}$, Leoncio D. Cruz-Gómez ${ }^{3}$, Mario A. Luna-Lamas² ${ }^{*}$, Italia N. Hinojosa-Olivares², \\ Regina R. Hernández-Vargas², Yesenia Delgado-Herrera² e Itzel A. Flores Hernández² \\ ${ }^{1}$ Anestesiología, Hospital General Zona N. ${ }^{0} 13$, Instituto Mexicano del Seguro Social, Matamoros; ${ }^{2}$ Facultad de Medicina, Instituto de Ciencias y \\ Estudios Superiores de Tamaulipas, Campus Matamoros; ${ }^{3}$ Dirección de Investigación, Instituto de Ciencias y Estudios Superiores de Tamaulipas, \\ Campus Tampico. Tamaulipas, México
}

\section{Resumen}

Objetivo: Demostrar que la práctica con simuladores es efectiva para que los alumnos de Medicina desarrollen la agilidad necesaria al momento de realizar una intubación endotraqueal. Material y método: Se llevó a cabo un estudio observacional simple ciego en el cual participaron 64 alumnos de Medicina del Instituto de Ciencias y Estudios Superiores de Tamaulipas Campus Matamoros 2001 de los grados de sexto y octavo semestre. En el estudio se evaluó el tiempo que les toma a los alumnos realizar una secuencia rápida de intubación en simuladores. Resultados: De acuerdo con los resultados, el $6.3 \%$ de los alumnos de sexto semestre logró realizar la técnica de intubación en menos de un minuto en comparación con los alumnos de octavo semestre, de los cuales el $78 \%$ realizó la técnica con éxito en menos de un minuto, ambos grupos en el primer registro de tiempo. En el segundo registro de tiempo los alumnos de sexto semestre mejoraron notablemente sus intervalos de tiempo, realizando la técnica con éxito el $90.6 \%$ en menos de un minuto y el $84 \%$ de los alumnos de octavo semestre en menos de un minuto. Conclusión: Por lo anterior podemos concluir que la práctica de intubación endotraqueal bajo simulación posterior al aprendizaje teórico es indispensable para adquirir habilidades manuales en cuanto a atención hospitalaria con pacientes reales se requiera.

Palabras clave: Intubación endotraqueal. Tiempo. Práctica. Vía aérea. Simulador.

\section{Practical method and time of endotracheal intubation among medical surgeon students}

\section{Abstract}

Objective: To demonstrate that the practice with simulators is effective for medical students to develop the necessary agility when performing endotracheal intubation. Material and method: A simple blind observational study was carried out in which 64 medical students participated in the ICEST Campus Matamoros 2001 of the sixth and eighth semester grades. In the study, the time it takes students to perform a rapid sequence of intubation in simulators was evaluated. Results: According to the results, $6.3 \%$ of students in the sixth semester managed to perform the intubation technique in less than a minute compared to the students in the eighth semester, of which $78 \%$ performed the technique successfully in less than a minute, both groups in the first time record. In the second time register, the students of the sixth semester improved their time intervals significantly by performing the technique successfully $90.6 \%$ in less than one minute, and $84 \%$ of the students in the

\section{Correspondencia:}

*Mario A. Luna-Lamas

E-mail: arthur2244@ hotmail.com
Disponible en internet: 16-04-2021

Fecha de recepción: 31-08-2020

Fecha de aceptación: 17-02-2021 DOI: $10.24875 /$ REIE.20000040
Rev Educ Investig Emer. 2021;3(1):3-8 www.medicinadeemergencias.com

2604-6520 (c) 2021 Sociedad Mexicana de Medicina de Emergencias, AC. Publicado por Permanyer México SA de CV. Este es un artículo open access bajo la licencia CC BY-NC-ND (http://creativecommons.org/licenses/by-nc-nd/4.0/). 
eighth semester in less than one minute. Conclusion: From the above we can conclude that the practice of endotracheal intubation under simulation after theoretical learning is essential to acquire manual skills in terms of hospital care with real patients is required.

Key words: Endotracheal intubation. Time. Practice. Airway. Simulator.

\section{Introducción}

La vía aérea es una de las más altas prioridades en un paciente críticamente enfermo ${ }^{1}$. Los primeros registros de abordaje de la vía aérea se hallan en tabletas egipcias que datan del 3600 a.C. En 1858, Eugene Bouchut, un pediatra francés, desarrolló la técnica de intubación orotraqueal no quirúrgica a ciegas: creó un tubo de metal para permitir la respiración evitando la obstrucción que las pseudomembranas de la difteria producían en la laringe. Su técnica se presentó el 18 de septiembre de 1858 en la conferencia de la Academia Francesa de Ciencias enfrentando la fuerte oposición del cirujano francés Armand Trousseau, quien promovía la traqueotomía en casos de obstrucción de la vía aérea².

Según el Consenso Internacional de Resucitación Cardiopulmonar y Atención Cardiovascular de Emergencia del 2005, ¿cuándo intubar? es una de las preguntas que más teme plantearse y resolver un profesional de la salud. En general, los pacientes en los que se sospecha que no podrán mantener la vía aérea protegida deben ser intubados: Glasgow igual o menor de 9, hematoma sofocante en cuello, obstrucción de la vía aérea, trauma de la vía aérea o maxilofacial, paciente agitado que requiere sedación, trauma torácico con hipotensión, hipoxia posreanimación, paro cardiaco, estigma de quemadura de vía aérea, quemaduras extensas, trauma raquimedular cervical, choque severo, insuficiencia respiratoria e incapacidad para mantener la vía aérea permeable ${ }^{3,4}$.

Rapidez es sinónimo de agilidad, ya que se requiere destreza y control del tiempo ${ }^{5}$. El aprendizaje de habilidades clínicas en "pacientes reales" no solamente pone en peligro la seguridad del paciente, también plantea múltiples cuestiones éticas. Esto requirió el desarrollo de laboratorios de habilidades en las facultades de Medicina donde los estudiantes aprenden y practican diversos aspectos del conocimiento y habilidades que imparten características positivas que mejoran la calidad del ambiente de aprendizaje ${ }^{6-8}$.

La adquisición de habilidades clínicas es un aspecto importante y un puente entre ganar conocimiento procesal y competencia clínica ${ }^{9}$. En la actualidad existe un amplio reconocimiento de que los médicos y los profesionales de la salud deben poseer una amplia gama de competencias para proveer atención de calidad para los pacientes. Estas van más allá del conocimiento médico o de las destrezas técnicas: dichas competencias se refieren al trabajo en equipo, liderazgo, profesionalismo, destrezas de relación interpersonal y de comunicación, toma de decisiones y algunas conductas que minimizan el riesgo de errores médicos y favorecen la seguridad del paciente ${ }^{10}$.

Los laboratorios de habilidades ofrecen un ambiente de capacitación para «perdonar errores» y los estudios han demostrado que dicha capacitación mejora las habilidades de procedimiento no solo en los novatos, sino también en los expertos ${ }^{11}$. Mediante la simulación se certifican ciertas habilidades y destrezas que se requieren en la profesión con el fin de promover una cultura de seguridad ${ }^{12}$.

La era moderna de la simulación médica tiene su origen en la segunda mitad del siglo xx, en la cual se identifican tres movimientos que impulsaron su desarrollo. El primero de ellos se inicia con la obra de Asmund Laerdal, quien en conjunto con médicos anestesiólogos y una fábrica de juguetes desarrolló un modelo de reanimación cardiopulmonar al que llamó «Resusci Anne»13,14.

El segundo movimiento está asociado con la simulación moderna y concierne al desarrollo de simuladores dedicados a reproducir de manera más precisa las características humanas de los pacientes con la creación del simulador SIMone ${ }^{\mathrm{TM}}$, desarrollado por Abrahamson y Denson a finales de la década de 1960 en la Universidad de Harvard ${ }^{14}$.

El tercer movimiento ha sido la reforma educativa mundial, la cual inició a finales del siglo pasado y continúa actualmente. Uno de los pilares de esta reforma es la búsqueda de nuevas estrategias de enseñanza aplicando las nuevas tecnologías, logrando un aprendizaje de habilidades clínicas y de comunicación, entrenamiento y formación en pregrado, posgrado y en educación médica continua ${ }^{15-17}$.

En la actualidad, el uso de simuladores es una realidad gracias a avances en tecnología y a la sistematización de aspectos relevantes de la teoría de la educación médica ${ }^{18}$. El marco teórico y conceptual de la simulación médica está centrado en el concepto de competencias, 
las cuales se definen como el conjunto de actitudes, destrezas, habilidades y conocimientos requeridos para realizar con calidad la labor profesional10,19.

Los alumnos de pregrado entrenados con simulación han demostrado menor estrés, mayor seguridad y mejor disposición a realizar ciertos procedimientos. Asimismo, se ha evidenciado una mejor disposición de los pacientes a ser atendidos por estudiantes cuando estos han sido entrenados previamente con simulación ${ }^{20-23}$. El desarrollo de la simulación como estrategia formativa ha dado lugar a un amplio conjunto de técnicas específicas como la asignación de roles, consistente en la representación de una situación social problemática que hay que asumir por medio de la recreación personal, mediante la representación de un papel distinto del que se juega en la vida ordinaria, estimula la implicación y la participación personal, da lugar a una expresión emocional abierta que facilita el diálogo, haciéndolo más fluido y positivo ${ }^{24,25}$.

La simulación es una herramienta que sirve para favorecer la realización de las mejores prácticas clínicas. Esta puede ser utilizada como objeto de investigación para validar su eficacia como herramienta educativa o puede ser utilizada para evaluar el desempeño del médico en un ambiente seguro y controlado, el cual asemeja al ambiente clínico habitual ${ }^{26}$.

La enseñanza del manejo de los pacientes en el departamento de urgencias siempre ha constituido un reto para el docente, ya que enseñar sobre un ser humano críticamente enfermo y permitir maniobras de los estudiantes en estos pacientes no solo es difícil, sino que se contrapone en forma directa con la norma de «no hacer daño", y está en contra del principio ético de «no maleficencia». Por lo anterior, la simulación no solo se convierte en una excelente herramienta de adiestramiento, sino en un apoyo ético para el paciente crítico y su familia. Se destaca la simulación en el prioritario y muchas veces complicado manejo de la vía aérea ${ }^{27,28}$.

\section{Material y método}

Se realizó un estudio observacional simple ciego con la participación de 64 alumnos de sexto y octavo semestre de la carrera de Medicina del Instituto de Ciencias y Estudios Superiores de Tamaulipas (ICEST) Campus Matamoros 2001, quienes previamente firmaron un consentimiento en el cual se les hacía mención de que sus datos serían utilizados solamente con fines de investigación.

El objetivo general de la investigación fue demostrar que la práctica con simuladores es efectiva para que los alumnos desarrollen la agilidad necesaria al momento de realizar una intubación endotraqueal; y dentro de los objetivos específicos se encuentran: a) comparar el tiempo que les toma realizar una secuencia rápida de intubación a los alumnos, b) fomentar una cultura de seguridad y confianza en los alumnos al momento de realizar el procedimiento de intubación endotraqueal, y c) promover el uso continuo del Centro de Enseñanza por Simuladores entre docentes y alumnos de la facultad de Medicina.

Los criterios de inclusión para el estudio fueron seleccionar a los alumnos inscritos en la facultad de medicina ICEST que cursaran el sexto y octavo semestre de la carrera, se excluyeron todos aquellos alumnos que no cumplieran con las características anteriores y se eliminaron a aquellos que no aceptaron participar en el estudio. En el estudio se evaluó el tiempo que les tomó a los alumnos realizar una secuencia rápida de intubación en simuladores utilizando el equipo para intubación del Centro de Enseñanza por Simuladores de la Facultad de Medicina ICEST, que incluye los simuladores SimMan 3G, Nasco Life/form, CPARLENE Life/form's, SimJunior y Resusci Anne. El material utilizado fueron tres laringoscopios con hoja de Machintoch del número 3, bolsa-válvula-mascarilla, guantes, tres tubos endotraqueales del número 7.5 con globo, tres guías, cronómetros y tablas para registrar los tiempos de intubación. Se realizaron dos registros de tiempo, el primero para evaluar cómo realizan la práctica con conocimiento teórico de la técnica y sin conocimiento de la práctica, y el segundo marcaje fue posterior a la explicación de la práctica.

Al momento de realizar el primer registro los alumnos de sexto semestre solo tenían conocimiento teórico de la técnica, sin haber realizado aún la práctica, mientras que los alumnos de octavo semestre ya tenían conocimiento teórico y práctico de la técnica. La variable dependiente utilizada en el estudio fue el tiempo, el cual se define como una magnitud física que permite ordenar la secuencia de los sucesos y cuya unidad en el sistema internacional es el segundo ${ }^{29}$. La variable independiente fue la intubación endotraqueal definida como una técnica utilizada para asegurar una vía aérea permeable ${ }^{30}$.

\section{Resultados}

De acuerdo con los resultados obtenidos en el primer registro de tiempo, representado en la figura 1, el 6.3\% de los alumnos de sexto semestre realizó la técnica de intubación de manera exitosa en menos de un minuto, mientras que el $62.5 \%$ de los alumnos tardó de uno a dos minutos en desarrollarla, y solo un $3 \%$ pudo desarrollar la técnica en un lapso de siete a ocho minutos. 


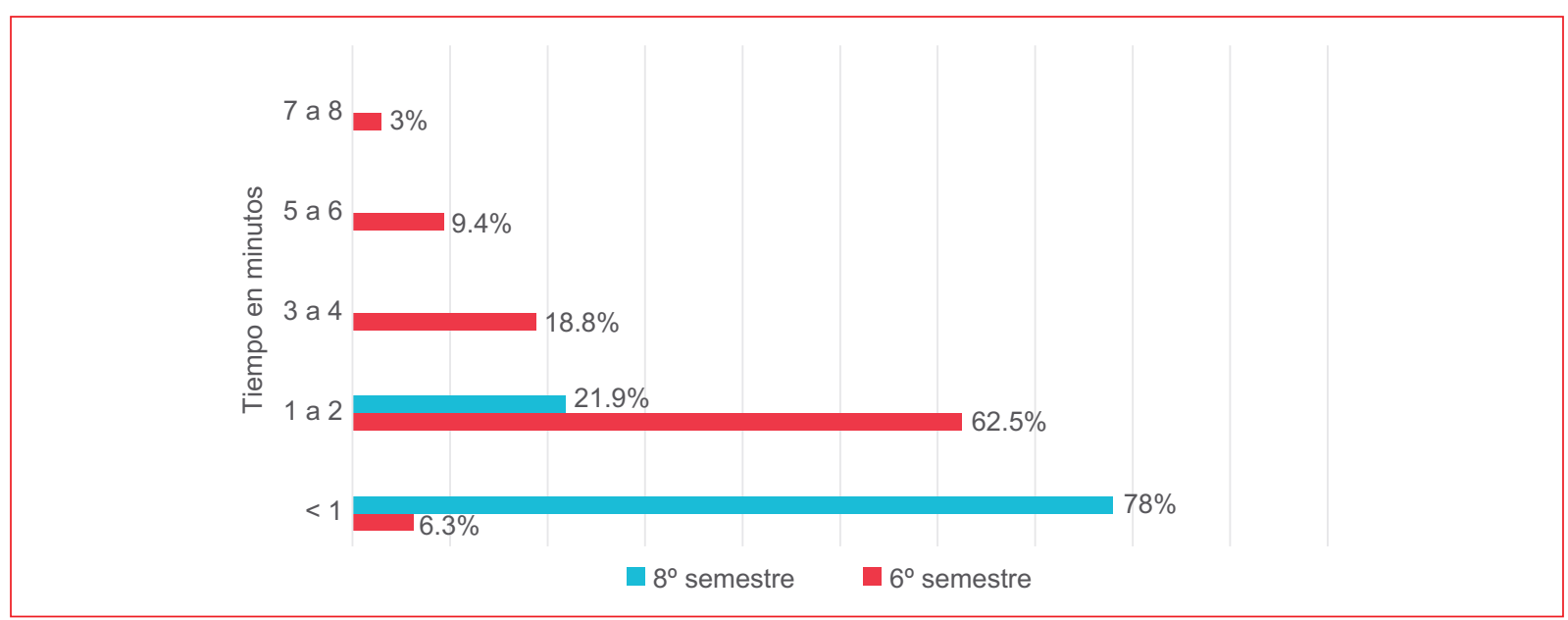

Figura 1. Comparación del primer registro de tiempo entre los alumnos de $6^{\circ}$ y $8^{\circ}$ semestre.

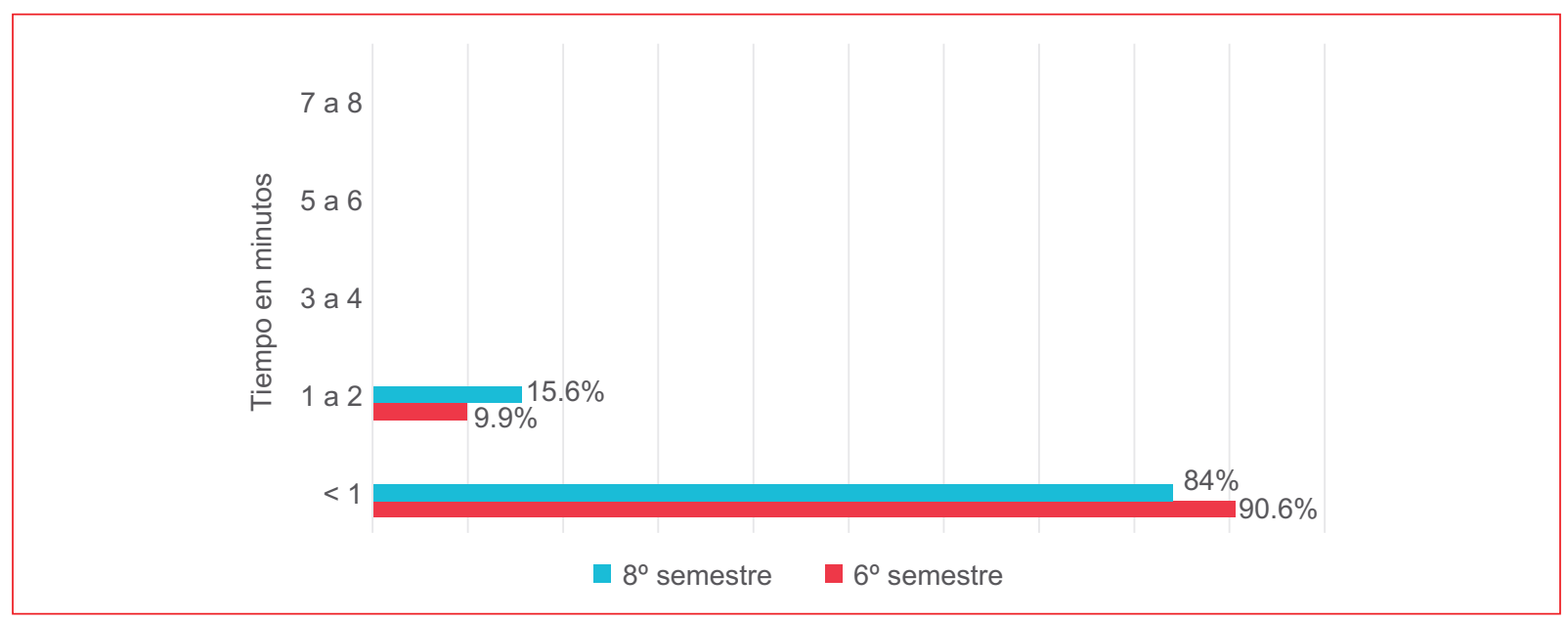

Figura 2. Comparación del segundo registro de tiempo entre los alumnos de $6^{\circ}$ y $8^{\circ}$ semestre.

Los alumnos de octavo semestre mostraron un intervalo de tiempo menor, el cual fue de un minuto en el $78 \%$ de los participantes, y el $21.9 \%$ de los alumnos realizó la técnica de uno a dos minutos. En el segundo registro de tiempo, que se muestra en la figura 2, los alumnos de sexto semestre mostraron una notable mejoría en cuanto al desarrollo de la técnica, obteniendo resultados del $90.6 \%$ en menos de un minuto y el $9.9 \%$ de uno a dos minutos. Por otra parte, el $84 \%$ de los alumnos de octavo semestre realizó la técnica en menos de un minuto y el $15.6 \%$ de uno a dos minutos.

Como se observa en las gráficas, la destreza de los alumnos para realizar una secuencia rápida de intubación mejoró de manera significativa, esto debido a que en el primer registro de tiempo los alumnos de sexto semestre solamente poseían conocimiento teórico de la técnica de intubación, lo que demuestra que la práctica resulta útil para desarrollar las habilidades clínicas. En cuanto a los alumnos de octavo semestre, ambos registros evidencian que su entrenamiento teórico-práctico de un año anterior a la realización del estudio les favoreció para realizar la técnica en un lapso menor de tiempo frente a sus compañeros de sexto semestre.

Ha quedado plenamente demostrado que cuando el estudiante tiene la competencia del saber, conoce la anatomía de la vía aérea superior, conoce y sabe clasificar las valvas para intubación y realiza esta en el maniquí, supera el miedo, es cuidadoso y tiene mayor certeza para realizar la maniobra. Se ha demostrado que residentes y estudiantes que utilizan los modelos para intubación orotraqueal logran la intubación en 
forma tan certera y rápida como los residentes experimentados de años superiores ${ }^{31,32}$.

\section{Discusión}

La gran mayoría de los programas en educación médica ha empleado la enseñanza basada en la resolución de problemas mediante casos clínicos. Se han desarrollado programas que incluso se pueden utilizar a distancia, como los de Shaw, et al., en el cual mediante casos clínicos se somete al estudiante a diferentes contextos en los cuales él debe dar las soluciones coherentes ${ }^{33}$. Utilizando la simulación podemos hacer que el estudiante desarrolle, paso a paso, con los modelos las maniobras específicas que realizaría si se enfrentara al «caso clínico» ${ }^{34,35}$. La técnica de simulación por sí sola puede convertirse en un actuar, sin contextualizar, por lo tanto, se recomienda que la simulación pedagógica sea la suma de: simulación (actuar) más la resolución de casos clínicos (razonamiento critico), unidos a la evaluación clínica objetiva y estructurada ${ }^{36-39}$. La intubación orotraqueal se considera uno de los procedimientos de mayor complejidad para el aprendizaje, se ha descrito una lenta curva de aprendizaje en diferentes estudios ${ }^{40-42}$.

Es un procedimiento que todos los médicos deberían ser capaces de realizar en situaciones de emergencia, $y$, por tanto, es indispensable que existan programas de entrenamiento bajo simulación que le permitan al alumno desarrollar la habilidad para realizarla. Habitualmente se necesitan entre 41 y 57 intubaciones para conseguir un $90 \%$ de éxito ${ }^{43}$. Este número se alcanza fácilmente durante la residencia de anestesiología y se adquiere con más rapidez en simuladores de pacientes ${ }^{44}$, sin embargo, no se produce en aquellos otros profesionales de la salud que se enfrentan con la necesidad de realizar una intubación orotraqueal. El uso de instrumentos y pautas de chequeo permiten establecer el logro de objetivos curriculares integrando los conocimientos teóricos y prácticos, con competencias actitudinales como control de la situación y liderazgo del alumno $45-47$. En nuestro estudio se demostró que la práctica aumenta la agilidad, la seguridad y la confianza en los alumnos, lo cual les permite realizar la técnica en el menor tiempo posible.

\section{Conclusión}

Por lo anterior podemos concluir que la práctica de intubación endotraqueal bajo simulación posterior al aprendizaje teórico es indispensable para adquirir habilidades manuales en cuanto a atención hospitalaria con pacientes reales se requiera, ya que observamos notable mejoría en cuestión de tiempo y de eficacia de la técnica, puesto que no hubo ninguna intubación fallida entre los 32 alumnos de sexto semestre. De acuerdo con los resultados en los alumnos un año después de recibir su adiestramiento teórico-práctico, se demuestra que siguen teniendo la habilidad aprendida y mejorando el tiempo de la técnica, sin embargo, presentaron tres intubaciones en segundo intento. Teniendo los anteriores resultados en alumnos de primera vez y de un año posterior a la práctica se podrían realizar mejorar en cuanto a una mayor eficacia de la técnica tomando como parámetro siguiente prácticas bajo simulación en los semestres posteriores antes de su egreso de la facultad. Hay que recordar que esta técnica se utiliza principalmente para maniobras de reanimación cardiopulmonar avanzadas intrahospitalarias.

\section{Agradecimientos}

Agradecemos al Dr. Daniel Ávalos Armenta, Coordinador del Centro de Enseñanza por Simuladores, quien nos bridó las facilidades para la realización de esta investigación; al Dr. Alberto Juan Salazar Vizuet, Secretario Académico de nuestra Facultad, por alentarnos a que nuestro estudio pudiera ser publicado; al Dr. Fermín Rosagaray Luevano, Director de la Facultad, por su incansable labor por mejorar la calidad de los alumnos egresados de nuestra casa de estudios, y a todos los coordinadores, docentes y compañeros, quienes siempre creyeron en nuestro trabajo como equipo.

\section{Financiamiento}

La presente investigación no ha recibido ninguna beca específica de agencias de los sectores público, comercial o sin ánimo de lucro.

\section{Conflicto de intereses}

Los autores de este artículo declaran no tener conflicto de intereses alguno.

\section{Responsabilidades éticas}

Protección de personas y animales. Los autores declaran que para esta investigación no se han realizado experimentos en seres humanos ni en animales. 
Confidencialidad de los datos. Los autores declaran que han seguido los protocolos de su centro de trabajo sobre la publicación de datos de pacientes.

Derecho a la privacidad y consentimiento informado. Los autores declaran que en este artículo no aparecen datos de pacientes

\section{Bibliografía}

1. Tse JC, Rim EB, Hussain A. Predicting difficult endotracheal intubation in surgical patients scheduled for general anesthesia: A prospective blind study. Anesth Analg.1995;81:254-8.

2. Trousseau A. Du tubage de la glotte et de la trachéotomie. Rapport fait à l'Académie Impériale de Médecine le 2 Novembre 1858. País: Baillière et Fils; 1858.

3. 2005 International consensus on cardiopulmonary resuscitation (CPR) and emergency cardiovascular care (ECC) science with treatment recommendations section 2: Stroke and first aid. Circulation. 2005;112:III-109.

4. Handley AJ, Koster R, Monsieurs K, Perkins GD, Davies S, Bossaert L, et al. European Resuscitation Council guidelines for resuscitation 2005 Section 2. Adult basic life support and use of automated external defibrillators. Resuscitation. 2005;67(Suppl 1):S7-S23.

5. Calder I, Pearce A. Core topics in airway management. Cambridge University Press; 2005. pp. 35-42.

6. Moon MR, Hughes MT, Chen JY, Khaira K, Lipsett P, Carrese JA. Ethics skills laboratory experience for surgery interns. J Surg Educ. 2014 71:829-38.

7. McGaghie WC, Issenberg SB, Petrusa ER, Scalese RJ. A critical review of simulation-based medical education research: 2003-2009. Med Educ. 2010;44:50-63.

8. Al-Elq AH. Medicine and clinical skills laboratories. J Family Community Med. 2007;14:59-63.

9. Lehmann R, Bosse HM, Simon A, Nikendei C, Huwendiek S. An innovative blended learning approach using virtual patients as preparation for skills laboratory training: perceptions of students and tutors. BMC Med Educ. 2013;13:23

10. Epstein RM, Hundert EM. Defining and assessing professional competence. JAMA. 2002:287:226-35.

11. Al-Yousuf $\mathrm{NH}$. The clinical skills laboratory as a learning tool for medical students and health professionals. Saudi Med J. 2004;25:549-51.

12. Bond W, Khun G, Binstadt E, Quirk M, Wu T, Tews M, et al. The use of simulation in the development of individual cognitive expertise in emergency medicine. Acad Emer Med. 2008;15:1037-45.

13. Bradley P. History of simulation in medical education and possible future directions. Med Educ. 2006;40(3):254-62.

14. Rosen KR. The history of medical simulation. J Crit Care. 2008;23(2):157-66.

15. Gordon MS. Cardiology patient simulator. Development of an animated manikin to teach cardiovascular disease. Am J Cardiol. 1974; 34(3):350-5.

16. Satava RM. Historical review of surgical simulation a personal perspective. World J Surg. 2008;32(2):141-8.

17. Ziv A, Wolpe PR, Small SD, Glick S. Simulation based medical education: an ethical imperative. Acad Med. 2003;78(8):783-8.

18. McLaughlin S, Fitch M, Goyal D, Hayden E, Kauh CY, Laack TA, et al. Simulation in Graduate Medical Education 2008: A review for emergency medicine. Acad Emerg Med 2008;15:1117-29.

19. Fox-Robichaud AE, Nimmo GR. Education and simulation techniques for improving reliability of care. Curr Opin Crit Care. 2007;13:737-41.

20. Okuda Y, Quinones J. The use of simulation in the education of emergency care providers for cardiac emergencies. Int $\mathrm{J}$ Emerg Med. 2008;1(2):73-7.

21. Issenberg SB, Gordon MS, Greber AA. Bedside cardiology skills training for the osteopathic internist using simulation technology. J Am Osteopath Assoc. 2003;103(12):603-7.
22. Issenberg SM, McGaghie WC, Gordon DL, Symes S, Petrusa ER, Hart IR, et al. Effectiveness of a cardiology review course for internal medicine residents using simulation technology and deliberate practice. Teach Learn Med. 2002;14(4):223-8.

23. Van Sickle KR. The pretrained novice: using simulation-based training to improve learning in the operating room. Surg Innov. 2006;13(3):198-204.

24. Salas E, Diaz Granados D, Weaver S, King H. Does team training work? Principles for Health care. Acad Emerg Med. 2008;15:1002-9.

25. DeVita MA, Schaefer J, Lutz J, Dongilli T, Wang H. Improving medical crisis team performance. Crit Care Med. 2004;32(S2):S61-S65.

26. McGaghie W, Draycott T, Dunn W, Lopez CM, Stefanidis D. Evaluating the impact of simulation on translational patient outcomes. Simul Healthc. 2011;6(suppl):S42-S47.

27. Overly FL, Sudikoff SN, Shapiro MJ. High-fidelity medical simulation as an assessment tool for pediatric residents' airway management skills. Pediatr Emerg Care. 2007;23(1):11-5.

28. Gisondi M, Smith-Coggins R, Harter P, Soltysik R, Yarnold P. Assessment of resident professionalism using high-fidelity simulation of ethical dilemas. Acad Emerg Med. 11(9):931-937.

29. Real Academia Española. Diccionario de la lengua española. Edición Tricentenario. Actualización 2020 [Internet]. Real Academia Española [consultado: 20 del octubre del 2019]. Disponible en: https://dle.rae.es

30. Poveda Jaramillo R, Dueñas Castell C, Ortíz Ruíz G. Secuencia rápida de intubación en cuidados intensivos. Rev Colomb Anestesiol. 2013;41(1):24-33.

31. Hodges D. Students benefit from airway simulator. Medical Post. 2003, 39(7):18.

32. Hall RE, Plant JR, Bands CJ, Wall AR, Kang J, Hall CA. Human patient simulation is effective for teaching paramedic students endotracheal intubation. Acad Emerg Med. 2005;12(9):850.

33. Shaw E, Ganeshan R, Johnson L, Millar D. Building a case for agent-assisted learning as a catalyst for curriculum reform in medical education. En: Lajoie SP, Vivet M, editores. Artificial intelligence in education. Ámsterdam, Países Bajos: IOS Press; 1999. pp. 509-16.

34. Greer J, Bull S. Workshop: Cognitive and social sciences foundations for medical education and training in the information age. Clin Invest Med. 2000;23(4):270-5

35. Greer J, McCalla G, Cooke J, Collins J, Kumar V, Bishop A, et al. The intelligent helpdesk: supporting peer help in a university course. En: Goettl BP, Halff HM, Redfield CL, Shute VJ, editores. Intelligent tutoring systems. Heidelberg: Springer-Verlag; 1998. pp. 494-503.

36. Davis MH. OSCE: the Dundee experience. Med Teach. 2003;25(3):255

37. Graceanne A. Uniformed services simulated and standardized patients in OSCEs: achievements and challenges 1992-2003. Med Teach. 2003; 25(3):262-70.

38. Bowen J. Educational strategies to promote clinical diagnostic reasoning. N Engl J Med. 2006;355(21):2217-25.

39. Morgan $\mathrm{P}$, Doreen M. Cost and resource implications of undergraduate simulator-based education. Can J Anaesth. 2001;48(8):827-8.

40. Konrad C, Schüpfer G, Wietlisbach M, Gerber H. Learning manual skills in anesthesiology: Is there a recommended number of cases for anesthetic procedures? Anesth Analg. 1998;86:635-9.

41. Rabitsch W, Staudinger T, Koestler WJ, Wulkersdorfer B, Urtubia R Frass $\mathrm{M}$, et al. Should there be a change in the teaching of airway management in the medical school curriculum? Resuscitation. 2005; 64:87-91.

42. Mulcaster JT, Mills J, Hung O, MacQuarrie K, Law A, Pytka S, et al. Laringoscopic intubation. Anesthesiology. 2003;98:23-7.

43. Bouchacourt JP, Castromán P. Evaluación del aprendizaje de la intubación orotraqueal mediante el método de la suma acumulativa (CuSum). Rev Esp Anestesiol Reanim. 2007:54:349-54.

44. Rall M, Gaba DM. Simuladores de pacientes. En: Miller RD, editor. Miller Anestesia. 6. ${ }^{a}$ edición. Philadelphia: Elsevier Churchill Livingstone; 2005. pp.3090-3093.

45. Bucley S, Colema NJ, Davison I. The educational effects of portfolios on undergraduate student learning: A Best Evidence Medical Education (BEME) systematic review. BEME Guide No. 11. Med Teach. 2009; 31:282-98.

46. Hunt EA, Shilko fski NA, Stavroudis TA, Nelson KL. Simulation: translation to improved team performance. Anesthesiol Clin. 2007;25:301-19.

47. Hart E, Owen $\mathrm{H}$. Errors and omissions in anesthesia: A pilot study using a pilot's checklist. Anesth Analg. 2005;101:246-50. 